
\title{
25 Research Soure \\ Mitigation of Scintillation Effects in WDM-FSO System Using Homodyne Detection
}

\section{Neha Rani}

Univesity Institute of Engineering and Technology, Panjab University, Chandigarh

Preeti Singh ( $\nabla$ preeti_singh@pu.ac.in )

Department of ECE,UIET, Panjab University, Chandigarh https://orcid.org/0000-0003-4680-6299

\section{Pardeep Kaur}

UIET: University Institute of Engineering and Technology, Panjab University, Chandigarh

\section{Research Article}

Keywords: FSO, WDM, Homodyne detection, Multibeam, Single beam, Scintillation

Posted Date: April 15th, 2021

DOl: https://doi.org/10.21203/rs.3.rs-372939/v1

License: (1) This work is licensed under a Creative Commons Attribution 4.0 International License. Read Full License

Version of Record: A version of this preprint was published at Optik on December 1st, 2021. See the published version at https://doi.org/10.1016/j.ijleo.2021.168165. 


\section{Abstract}

Free Space Optics is a line of sight communication which transmits a very narrow beam width. FSO offers high bandwidth and higher data rate up to 10Gbps. FSO units are transportable, compact and are easy to redeploy. With so many advantages of FSO there exists serious limitations of atmospheric effects which makes it inefficient for long reach transmission. Environment factors act as barriers in efficient utilization of FSO technology. A major issue in FSO links is the turbulence induced fading which severely deteriorates the link performance, hence need to be alleviated for the efficient use of this rapidly emerging technology. Various researches have been carried out in FSO field to minimize the effect of turbulence induced scintillation. One such technique has been employed in this paper in which coherent based homodyne detection is utilized in conjunction with multibeam technique in WDM-FSO system with aim to reduce the scintillation effect. The performance comparison of the homodyne detection and direct detection in terms of bit error rate, Q- factor, and eye diagram has been explored for the scintillating effect.

\section{Introduction}

The growing interest to seek for high speed and pervasive broadband remote access has prodded massive development in internet traffic demand. Subsequently, ten folds rise in data traffic after every five years, relates to a terrific development in general traffic volume. Supplementing the current RF solutions, FSO has proven to be a moderate and appealing high information rate innovation and is ready to turn into a promising contender to determine the current "last mile" network problem. It is for the most part because of the immensely attractive highlights of FSO innovation like low power utilization, diminished interference, high adaptability and re-configurability and a high level of security [1]. Major issue in FSO links is the turbulence induced fading which severely deteriorates the performance of FSO system. Various techniques have been developed and are still being carried out in order to limit the effect of atmospheric losses and to boost up the quality of signal transmission. Numerous techniques are available to conquer the essential drawback of attenuation. However, one of the encumbrance is atmospheric scintillation, which can be the source of intensity blurring in FSO systems thus creating this invention badly arranged for applications demanding high accessibility. Some of the techniques to raise the efficiency of FSO communication have been reviewed in the following section.

\section{Related Study}

Deep fades owing to scintillation with high probabilities of errors, can substantially dam-age system efficiency overall. Various kinds of mitigation methods such as diversity [2], [3], aperture averaging [4], [5], Erbium-doped fiber amplifier (EDFA) [6] are used to enhance FSO efficiency and reliability for all weather conditions. FSO can attain greater reliability and capability. Key advances in technology such as orthogonal frequency division (OFDM) [7] and wavelength-division multiplexing (WDM) [8] have extended the FSO system capacity. Due to outstanding immunity to multipath fading resistance and frequency selective fading, OFDM has been widely used in wireless communication over many years. There has 
recently been a new optical equivalent known as Coherent-OFDM. In this scheme, the benefits of coherent detection and OFDM are combined. Wavelength division multiplexing is another technique which is used extensively to increase FSO capacity at very small channel spacing and elevated information rates. The WDM (Wavelength Multiplexing Division) is adaptable and reliable which can provide wide coverage by supporting the large number of users. This system is mainly limited by atmospheric turbulence that impedes the general system efficiency. Many parameters such as bit rate, failure rate, etc. get affected. The scheme can give better outcomes if appropriate modifications are made at different phases. One way is to use the virtual local oscillator in the receiver segment using a coherent detection technique. Detection techniques are categorized primarily as a direct detection and coherent detection. A single photodiode is used with the direct detection system whereas an optical mixing principle is used in a coherent detection system. The comparative analysis in terms of BER of direct detection FSO and coherent detection FSO communication schemes is done in [9] under the weak atmospheric turbulence over a log normal distributed channel. Results lead to the conclusion that BER of coherent detection is less when compared to the direct detection.

\section{Coherent Detection}

When WDM is combine with coherent detection, the advantages of these two potent methods are multipurpose. Coherent detection may be a promising technology to detect weak optical signal after its propagation. Coherent detection is a much more complex technique of detection compared to conventional intensity modulation and direct detection schemes (IM / DD), but can allow greater sensitivity to the receivers [10]. The coherent communication schemes allow a big amount of WDM optical channels to transmit with very tight frequency divisions, as outlined in [11]. The ability to select any specific channel by merely setting a local oscillator (LO) is another benefit of coherent detection. Coherent receivers with impressive characteristics can be regarded as nearly an ideal optical filter. Coherent receivers can further be classified as homodyne and heterodyne receiver. Advances in laser technology for semiconductors has stimulated a renewed interest in the technology of both optical heterodyne and homodyne detection methods. The use of heterodyne technique at $\mathrm{THz}$ has been driven by two features. First and the foremost, since the signal carrying the useful data is down converted, it is possible to use low frequency amplifiers. Even at very high frequency when direct amplification is not feasible owing to the absence of high-speed amplifiers, this enables the use of heterodyne receivers. Second, by selecting a detection bandwidth comparable to the signal bandwidth, the noise can be decreased.

It has been discovered that the efficiency of heterodyne detection considerably deteriorates under conditions of atmospheric turbulence. A tutorial comparison of optical heterodyne and homodyne techniques and a qualitative assessment of the effect of actual device properties on homodyne and heterodyne systems are presented in [12] which demonstrates that optical homodyne detection can offer a $3 \mathrm{~dB}$ performance improvement compared to optical heterodyne. Also, Homodyne systems have a maximum information rate limit of 5 to 6 times that of heterodyne systems. The effect of turbulent atmosphere such as scintillation and loss of power in the detector can further be decreased to larger 
extent using Multi-beam system [13] which is a form of spatial diversity. Diversity helps in alleviation of fading but in direct detection system diversity cannot be increased beyond an optimum value [14] as background noise comes into existence at higher level of diversity, so an optimum solution is use of coherent based homodyne detection. In contrast to diversity direct detection, Diversity coherent detection allow higher level of diversity without any increase background noise Wavelength division multiplexing (WDM) technique in conjunction with multibeam FSO offers many advantages by supporting high capacity transmissions.

\section{Proposed System}

Proposed Multibeam WDM FSO System is the one which combine the advantages of WDM, spatial diversity and homodyne detection so as to enhance the system under the scintillation effect as shown in Fig. 1. Wavelength Division Multiplexing is a technology of modulating multiple data streams on optical carriers of different wavelength which are sent through a single channel [15].

A multiplexer at the transmitter is used for multiplexing of different modulated carriers and a de multiplexer at receiver end used for performing the reverse operation. WDM offers multiplication of signal capacity. WDM technique when used in FSO is called as WDM-FSO system.

Wavelength division multiplexing (WDM) technique in conjunction with multibeam FSO offers many advantages by supporting high capacity transmissions. The effect of turbulent atmosphere such as scintillation and loss of power in the detector can be decreased to larger extent using Multibeam system which make use of spatially diverse transmitters [16]. In the present work, signal is detected taking the advantage of homodyne detection in order to improve the receiver sensitivity. This technique serve as a solution for reducing the scintillation effect [17]. Homodyne detection includes the merging of the received signal with the local oscillator signal (having identical frequency and phase as that of the signal received) prior to the photo detection. This detection is a moderately simple method of intensifying the photo current by rising the power of local oscillator. This detection delivers better SNR by expanding the local oscillator control.

\section{Simulation}

Single beam WDM-FSO (SB WDM-FSO) system and multibeam WDM-FSO (MB- WDM FSO) system are analyzed using the coherent based homodyne detection under the scintillation effect in the present work. SB WDM-FSO system and MB WDM-FSO systems employing direct detection at the receiver are the preexisting systems taken from the base paper [13]. Incorporating Homodyne detection, SB WDM-FSO system and MB WDM-FSO system are modified with the aim to reduce the effect of scintillation. This research was performed by using a turbulence "gamma-gamma" model [18], when turbulence ranges from moderate to strong.

\section{Single beam WDM FSO system (System I)}


SB WDM-FSO system using Homodyne detection has been designed in Optisystem environment as shown in Fig. 2. Transmitter section comprises pseudo-random bit sequence generator, Non Return to Zero (NRZ) electrical pulse generator, Continuous wave laser (CW), WDM MUX and Machzender modulator (MZM).

The receiver section of the system includes WDM-DEMUX followed by a selector switch. Homodyne detection is used at the receiver for extraction of the information signal due to its superiority over the direct detection. Received signal is mixed with the signal generated by the local oscillator using an optical coupler. Photodiodes will recognize the interference of the received signal and local oscillator signal. Finally, the subtraction of the signals from two photodiodes is performed which result in output signal yielding large information. Resulting signal is filtered by the low pass Bessel filter in order to get the desired signal. Eye diagram analyzer is used for BER and Q factor measurements. Table 1 displays different parameters and values used for FSO system simulation.

Table 1

Simulation parameter values

\begin{tabular}{|ll|}
\hline PARAMETER & VALUES \\
\hline Laser wavelength & $1552 \mathrm{~nm}$ \\
\hline Laser power & $10 \mathrm{dBm}$ \\
\hline Data rate & $10 \mathrm{Gbps}$ \\
\hline Transmitter aperture & $15 \mathrm{~cm}$ \\
\hline Receiver aperture & $15 \mathrm{~cm}$ \\
\hline Attenuation & $0.065 \mathrm{~dB} / \mathrm{km}$ \\
\hline Beam divergence & $2 \mathrm{mrad}$ \\
\hline Geometric losses & YES \\
\hline
\end{tabular}

\section{Multibeam WDM-FSO System using Homodyne detection (System II)}

Figure 3 shows a Multibeam WDM-FSO System in which Homodyne detection is applied on the receiver section. The transmitter and the receiver portions of Multibeam WDM FSO system are identical to that of single beam WDM FSO system. A fork after the MZM modulator is used to get the four modulated signals; the setup is analogous to realizing four different transmitter apertures which transmit four mutually independent beams following particular ways. Multibeam technology follows the fact that when beam propagates through different paths, they encounter different amounts of attenuation.

\section{Results}

The analysis has been resolved in following sections. First section bears out the performance of $S B$ WDM-FSO system and MB WDM-FSO system using Homodyne detection under the scintillation effect, 
followed by the section demonstrating the comparative analysis of both systems along with the comparative analysis of preexisting Multibeam WDM-FSO system using direct detection with the proposed Multibeam WDM-FSO system using Homodyne detection and the last section optimizes the input power of the proposed system.

\section{Performance Analysis of SB WDM-FSO System and MB WDM-FSO System}

For Single beam WDM FSO system using Homodyne detection, refractive index structure parameter $C^{2}$ is taken as $10^{-13} \mathrm{~m}^{-2 / 3}$ which corresponds to the high turbulent conditions. When simulated for this value of high turbulence, maximum link distance achieved is $10 \mathrm{~km}$. Beyond the link range of $10 \mathrm{~km}$, the quality factor drops down from the acceptable value required for efficacious transmission i.e. around ' 6 ' and also BER rises above the acceptable error of $10^{-9}$. Q-factor and BER value obtained through the BER analyzer for $10 \mathrm{~km}$ is 6.13 and $4.01 \mathrm{e}-10$ respectively.

Multibeam WDM-FSO system uses laser power of $10 \mathrm{dBm}$. One of the key advantage of Multibeam technology used here is to lessen the effect of scintillation. Four beams of the system encounter different amount of scintillation depending on the value of $C^{2}$ The value of $C_{n}^{2}$ is taken as $10^{-13}, 10^{-14}$, $10^{-15}$ and $10^{-16} \mathrm{~m}^{-2 / 3}$ to represent different refractive indices effect due to different independent paths. This system can work efficiently up to $25 \mathrm{~km}$ with Q factor of 6.04 and BER of 7.27e-10 at data rate of 10Gbps. With further increase in distance, Q factor falls below the acceptable value for successful transmission.

\section{Comparative analysis of Single beam and Multibeam WDM FSO System using Homodyne detection}

Single beam and Multibeam WDM FSO system using Homodyne detection have been analysed in terms of Quality Factor and BER. The Quality Factor reduces and Bit Error Rate of the system rises with the rise in link length, thus degrading the system performance at higher link length. It tends to be observed from the graph in Fig. 4 that Q-factor lessen underneath the adequate worth when link length is increased beyond $10 \mathrm{~km}$ for the SB-WDM FSO system using Homodyne detection but for the Multibeam WDM FSO system using Homodyne detection it remains above the acceptable value ' 6 ' till $25 \mathrm{~km}$. Hence, acceptable transmission can be achieved up to longer link length for MB-WDM FSO system using Homodyne detection as compared to SB WDM -FSO system for the same input power requirement.

Figure 5 compares the eye diagram of Single beam and Multibeam WDM FSO system using Homodyne detection at link distance of $10 \mathrm{~km}$ with data rate of $10 \mathrm{Gbps}$ and input launch power of $10 \mathrm{dBm}$ under scintillation effect. The eye diagram of MB-WDM FSO system using Homodyne detection is wide open as compared to SB-WDM FSO system using Homodyne detection. The eye height of SB-WDM FSO system using Homodyne detection is seen from eye diagram is around $53 \mu \mathrm{m}$ whereas for Multibeam WDM FSO system using Homodyne detection is $386 \mu \mathrm{m}$, indicates high quality of received signal.

The comparison of both the system is given in Table 2. For practically same Q Factor and BER, the separation length of MB-WDM FSO framework utilizing Homodyne detection is more prominent than that 
of SB-WDM FSO framework utilizing Homodyne detection which point towards the more prominent productivity under the scintillation impact.

Table 2: Performance of SB WDM-FSO and MB WDM- FSO system using Homodyne detection

\begin{tabular}{|c|c|c|c|c|c|}
\hline System & $C_{n}^{2}\left(m^{-2 / 3}\right)$ & $\begin{array}{l}\text { Max range } \\
(\mathrm{km})\end{array}$ & $\begin{array}{l}\text { Min } \\
\text { BER }\end{array}$ & $\begin{array}{c}\mathrm{Q} \\
\text { factor }\end{array}$ & $\begin{array}{c}\text { Eye } \\
\text { height }\end{array}$ \\
\hline $\begin{array}{l}\text { SB WDM-FSO system using } \\
\text { Homodyne detection }\end{array}$ & $10^{-13}$ & $10 \mathrm{~km}$ & $\begin{array}{l}4.01 \mathrm{e}^{-} \\
10\end{array}$ & 6.13 & $53 \mu \mathrm{m}$ \\
\hline $\begin{array}{l}\text { MB WDM- FSO system using } \\
\text { Homodyne detection }\end{array}$ & $\begin{array}{c}10^{-13}, 10^{-14}, 10^{-15} \\
10^{-16}\end{array}$ & $25 \mathrm{~km}$ & $\begin{array}{l}7.27 \mathrm{e}- \\
10\end{array}$ & 6.04 & $386 \mu \mathrm{m}$ \\
\hline
\end{tabular}

\section{Comparative analysis of MB WDM-FSO system using Direct detection (preexisting) and MB WDM-FSO system using Homodyne detection (proposed).}

MB WDM-FSO system using direct detection has been designed in paper [13]. It has been observed that with laser power of $10 \mathrm{dBm}$, system can communicate up to $4.5 \mathrm{~km}$ with information rate of $10 \mathrm{Gbps}$ under the scintillation effect. MB WDM-FSO system using Homodyne detection is designed in the present work which can transmit up to $25 \mathrm{~km}$ under the turbulent atmosphere with the same data rate and at same input laser power.

Performance of MB WDM-FSO system using direct detection and newly designed MB WDM-FSO system using Homodyne detection is compared graphically in Fig. 6 and Fig. 7. Figure 6 compares the two systems in terms of $\mathrm{Q}$ factor variation with respect to the link distance and shows the difference in quality of received signal at various link distance. Graph illustrates that $\mathrm{Q}$ factor for the system with direct detection is acceptable up to a distance of $4.5 \mathrm{~km}$ and becomes zero at a distance of $8.5 \mathrm{~km}$ while for the system using Homodyne detection it remains above the acceptable range till $25 \mathrm{~km}$.

Also, both of these systems are compared using eye diagrams in Fig. 7. From the Fig. 7, it is clear that eye height of the system with Homodyne detection is far better than system with direct detection. For the system with direct detection, eye height is noted to be $11.5 \mu \mathrm{m}$ while for the Homodyne detection system eye height is $386 \mu \mathrm{m}$, this large difference in eye height at a distance of $4.5 \mathrm{~km}$ indicates the better quality of the signal reception under the scintillation effect.

The performance comparison of system using direct detection with the system using Homodyne detection has been tabulated in Table 3. The link range achieved for the different value of refractive indices for different path for the system using direct detection under the scintillation effect is $4.5 \mathrm{~km}$ with $\mathrm{Q}$ factor of 5.85 and for the system with Homodyne detection it is $25 \mathrm{~km}$ with Q factor of 6.04 .

Table 3: Comparison of Performance of Multibeam WDM-FSO using Direct detection with the Multibeam WDMFSO system using_Homodyne detection 


\begin{tabular}{|c|c|c|c|c|c|}
\hline System & $C_{n}^{2}\left(m^{-2 / 3}\right)$ & $\begin{array}{l}\text { Max range } \\
\quad(\mathrm{km})\end{array}$ & $\begin{array}{l}\text { Min } \\
\text { BER }\end{array}$ & $\begin{array}{c}\mathrm{Q} \\
\text { factor }\end{array}$ & $\begin{array}{c}\text { Eye } \\
\text { height }\end{array}$ \\
\hline $\begin{array}{l}\text { Multibeam WDM-FSO system using } \\
\text { Direct detection }\end{array}$ & $\begin{array}{l}10^{-13}, 10^{-14}, \\
10^{-15}, 10^{-16}\end{array}$ & $4.5 \mathrm{~km}$ & $\begin{array}{l}2.25 \mathrm{e}^{-} \\
009\end{array}$ & 5.85 & $11.5 \mu \mathrm{m}$ \\
\hline $\begin{array}{l}\text { Multibeam WDM- FSO system using } \\
\text { Homodyne detection }\end{array}$ & $\begin{array}{l}10^{-13}, 10^{-14} \\
10^{-15} 10^{-16}\end{array}$ & $25 \mathrm{~km}$ & $\begin{array}{l}7.27 e^{-} \\
10\end{array}$ & 6.04 & $386 \mu \mathrm{m}$ \\
\hline
\end{tabular}

In commercial FSO System, such a long distance is not the requirement as the performance of FSO system is limited by line of sight requirement at longer distance. Additionally, it requires tight pointing acquisition and tracking (PAT) mechanism for the effective trans- mission. Power can be optimized so as to advance the framework execution at shorter separation. Efforts are made to reduce the power requirement and its effect on link distance was observed.

\section{Power optimization of Multibeam WDM-FSO system using Homodyne detection under the effect of scintillation}

In the face of growing world-wide energy consumption, research communities have recognized the need to save energy in every possible manner. Minimizing the total power consumption of wireless networks is a well-recognized way to improve the energy efficiency and thus to have a hand in the reduction of worldwide energy consumption. The input launch power of an optical source is a very significant factor for communication researchers in designing free space optics communication systems. The high data rate transmission demands an increase in input launch power or equivalent enhancement in receiver sensitivity. However, increase in power endanger the safety of human beings and is harmful to human eyes if launched above the permissible limit. The proposed model provides a finest solution as far as power optimization is concerned.

In present work, power has been optimized for the Multibeam WDM-FSO system using Homodyne detection. The laser power used was $10 \mathrm{dBm}$ at the time of system design and it has been shown in previous result that link distance of $25 \mathrm{~km}$ can be achieved for an acceptable value of $\mathrm{Q}$ factor at this power level.

Power has been optimized in order to optimize the system performance at distance of $4.5 \mathrm{~km}$ with data rate of $10 \mathrm{Gbps}$ as shown in Fig. 8 . It is clear from the graph that the power of $-3 \mathrm{dBm}$ is sufficient to achieve a link distance of $4.5 \mathrm{~km}$. Quality factor at the distance of $4.5 \mathrm{~km}$ with input power launch of $-3 \mathrm{dBm}$ has been recorded as 6.60 .

Figure 9 demonstrates the eye diagram of Multibeam WDM-FSO system using Homodyne detection at a distance of $4.5 \mathrm{~km}$. Eye height of the system is observed as $13.9 \mu \mathrm{m}$. Further attempt was made to see the effect of power reduction on link distance. In order to optimize the power, feasible distance corresponding to various power values were recorded through simulation in Optisystem. When the power level was kept $-6 \mathrm{dBm}$, the Q factor achieved was 5.67 for distance of $3 \mathrm{~km}$. With decrease in the power level, link 
distance decreases. So corresponding to the $2 \mathrm{~dB}$ decrease in power, link distance is decreased by approx. $1 \mathrm{~km}$.

It can be concluded that for MB WDM-FSO system using coherent detection, power level around - 3dBm can serve the purpose. Reducing the power beyond this value reduces the link length and makes it unsuitable for successful transmission.

\section{Conclusion}

It has been observed that using Homodyne detection at the receiving node, performance of the existing WDM-FSO system is improved significantly. When Homodyne detection applies to single beam and multibeam WDM FSO system, MB WDM-FSO system gives better performance as compared to SB WDM FSO system under the scintillation effect. For a distance of $4.5 \mathrm{~km}$, laser power requirement for MB WDM-

FSO system using homodyne detection is reduced to $-3 \mathrm{dBm}$ as compared to MB WDM-FSO system using direct detection which required $10 \mathrm{dBm}$ power to operate at same distance.

\section{Declarations}

Funding: Not Applicable

Conflicts of interest/Competing interests: There is no conflict of interests in the present work.

Availability of data and material: The referred work is cited in the related study section.

Code availability: The research work is carried out using optisystem software as discussed in simulation section.

\section{References}

1. Malik, A., \& Singh, P., "Free Space Optics: Current Applications and Future Challenges," Int. J. Opt., vol. 2015, no. c, pp. 1-7, 2015.

2. Nistazakis, H. E., \& Tombras, G. S. "On the use of wavelength and time diversity in optical wireless communication systems over gamma-gamma turbulence channels," Opt. Laser Technol., 2012.

3. Navidpour, S. M., Uysal, M., \& Kavehrad, M. (2007). BER performance of free-space optical transmission with spatial diversity. IEEE Trans. Wirel. Commun., 6(8), 2813-2819.

4. Khalighi, M. A., Schwartz, N., Aitamer, N., \& Bourennane, S., "Fading reduction by aperture averaging and spatial diversity in optical wireless systems," J. Opt. Commun. Netw., vol. 1, no. 6, pp. 580-593, Nov. 2009.

5. Khalighi, M. A., Aitamer, N., Schwartz, N., \& Bourennane, S., "Turbulence mitigation by aperture averaging in wireless optical systems," in 2009 10th International Conference on Telecommunications, ConTEL 2009, 2009, pp. 59-66. 
6. Abtahi, M., \& Rusch, L. A., "Mitigating of scintillation noise in FSO communication links using saturated optical amplifiers," in Proceedings - IEEE Military Communications Conference MILCOM, 2007.

7. Chaudhary, S., Amphawan, A., \& Nisar, K. (2014). Realization of free space optics with OFDM under atmospheric turbulence. Optik (Stuttg)., 125(18), 5196-5198.

8. Sher, M. S. M., Khalil, I., Biswas, A., Rakib, R. B., \& Sayeed, M. A. (2019). WDM transmission for free space optics under different atmospheric conditions. Trends in Opto-Electro and Optical Communications, 4(1), 7-12.

9. Kumar, P., "Comparative analysis of BER performance for direct detection and coherent detection FSO communication systems," Proc. - 2015 5th Int. Conf. Commun. Syst. Netw. Technol. CSNT 2015, pp. 369-374, 2015.

10. Andrews, L. C., \& Phillips, R. L. "Laser Beam Propagation Laser Beam Propagation SECOND EDITION.".

11. Khoe, G.-D., "Coherent multicarrier lightwave technology for flexible capacity networks," IEEE Commun. Mag., vol. 32, no. 3, pp. 22-33, Aug. 2002.

12. Kazovsky, L. G., "H Journal of Optical Heterodyning Versus Optical Homodyning: A Comparison," vol. 6, no. 1965, pp. 18-24, 1985.

13. Grover, M., Singh, P., \& Kaur, P., "Mitigation of scintillation effects in WDM FSO system using multibeam technique," J. Telecommun. Inf. Technol., vol. 2017, no. 2, pp. 69-74, 2017.

14. Lee, E. J., \& Chan, V. W. S. "Power gain of homodyne detection over direct detection receivers for free space optical communication in the presence of interference," 2008 Conf. Quantum Electron. Laser Sci. Conf. Lasers Electro-Optics, CLEO/QELS, pp. 8-9, 2008.

15. Malik, A., \& Singh, P. (2014). Comparative Analysis of Point to Point FSO System Under Clear and Haze Weather Conditions. Wirel. Pers. Commun., 80(2), 483-492.

16. Tsiftsis, T. A., Sandalidis, H. G., Karagiannidis, G. K., \& Uysal, M. (Feb. 2009). Optical wireless links with spatial diversity over strong atmospheric turbulence channels. IEEE Trans. Wirel. Commun., 8(2), 951-957.

17. Sahota, J. K., \& Dhawan, D., "Reducing the effect of scintillation in FSO system using coherent based homodyne detection," Optik (Stuttg)., vol. 171, no. June, pp. 20-26, 2018.

18. Parikh, J., \& Jain, V. K., "Study on statistical models of atmospheric channel for FSO communication link," 2011 Nirma Univ. Int. Conf. Eng. Curr. Trends Technol. NUiCONE 2011 - Conf. Proc., pp. 1-7, 2011.

\section{Figures}




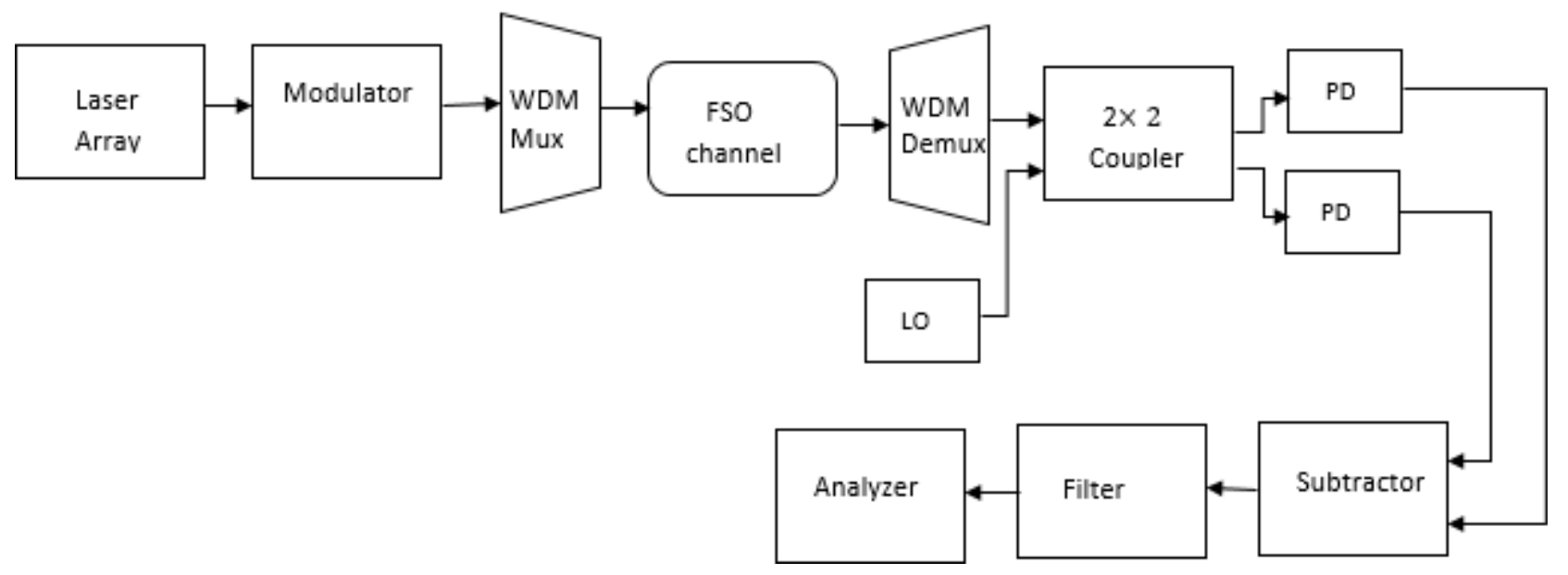

Figure 1

Multibeam WDM-FSO system using Homodyne detection
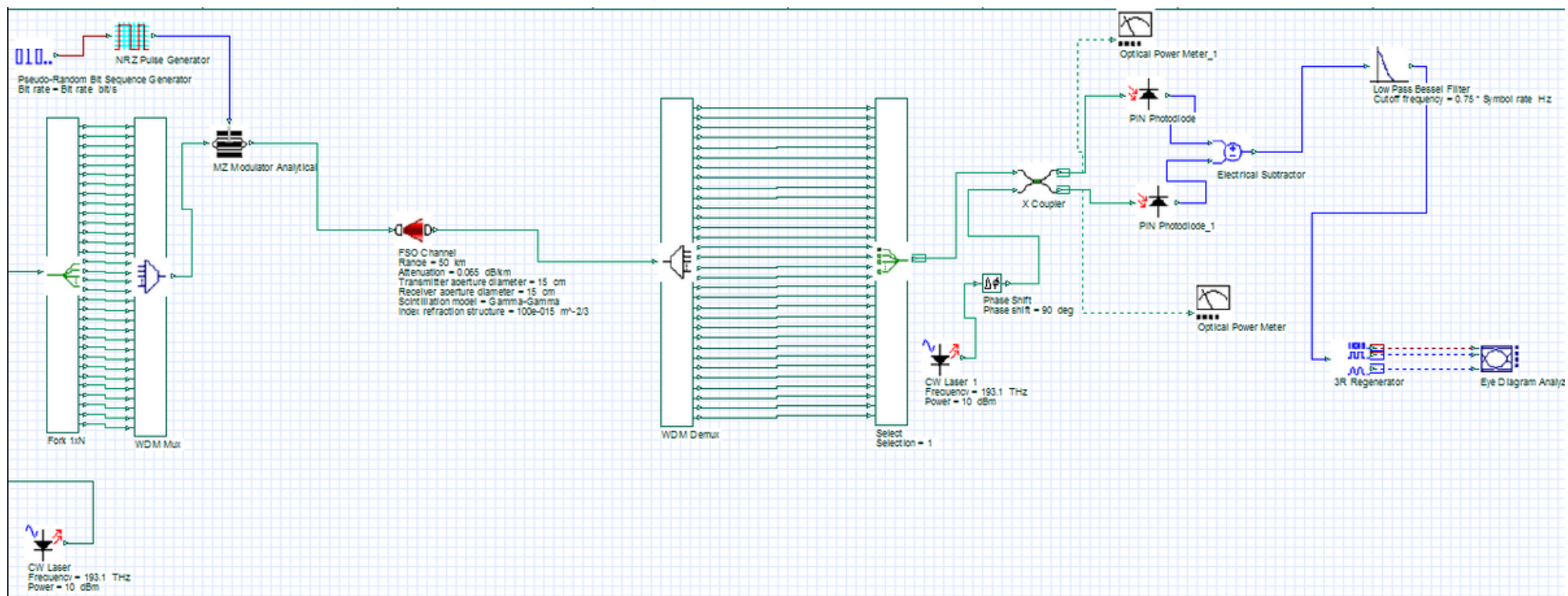

Figure 2

SB WDM-FSO system using homodyne detection 


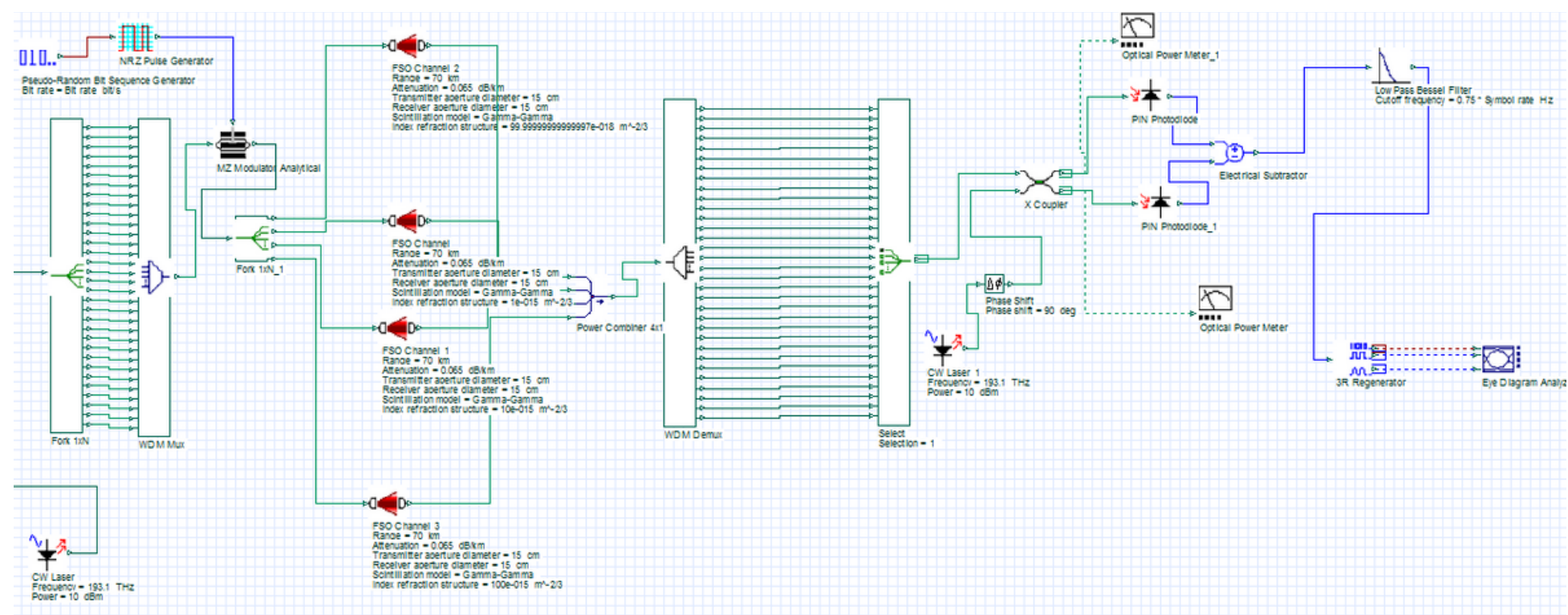

Figure 3

MB WDM-FSO system using homodyne detection

\section{Q-factor v/s Link distance}

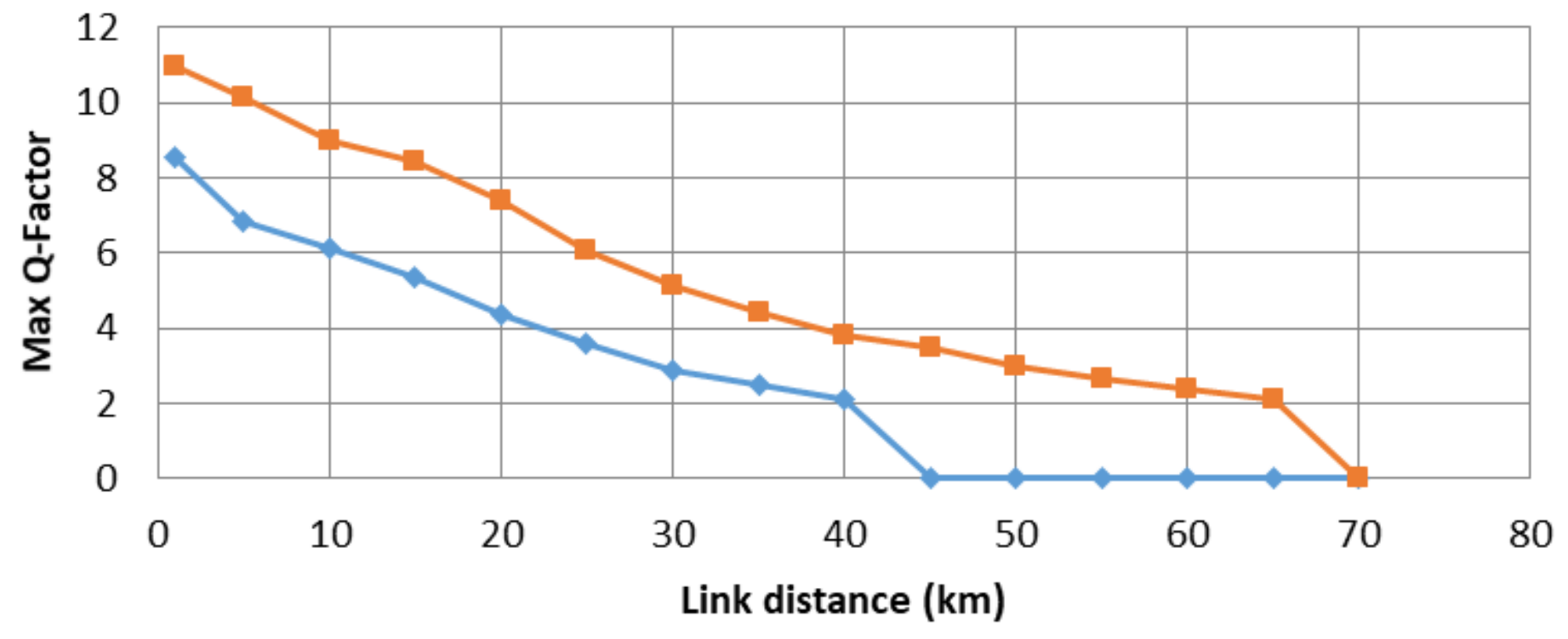

$\multimap$ SB-WDM FSO System using Homodyne Detection

- -MB WDM-FSO System using Homodyne detection

Figure 4

Comparison of SB-WDM and MB-WDM FSO system using Homodyne detection under scintillation effect 

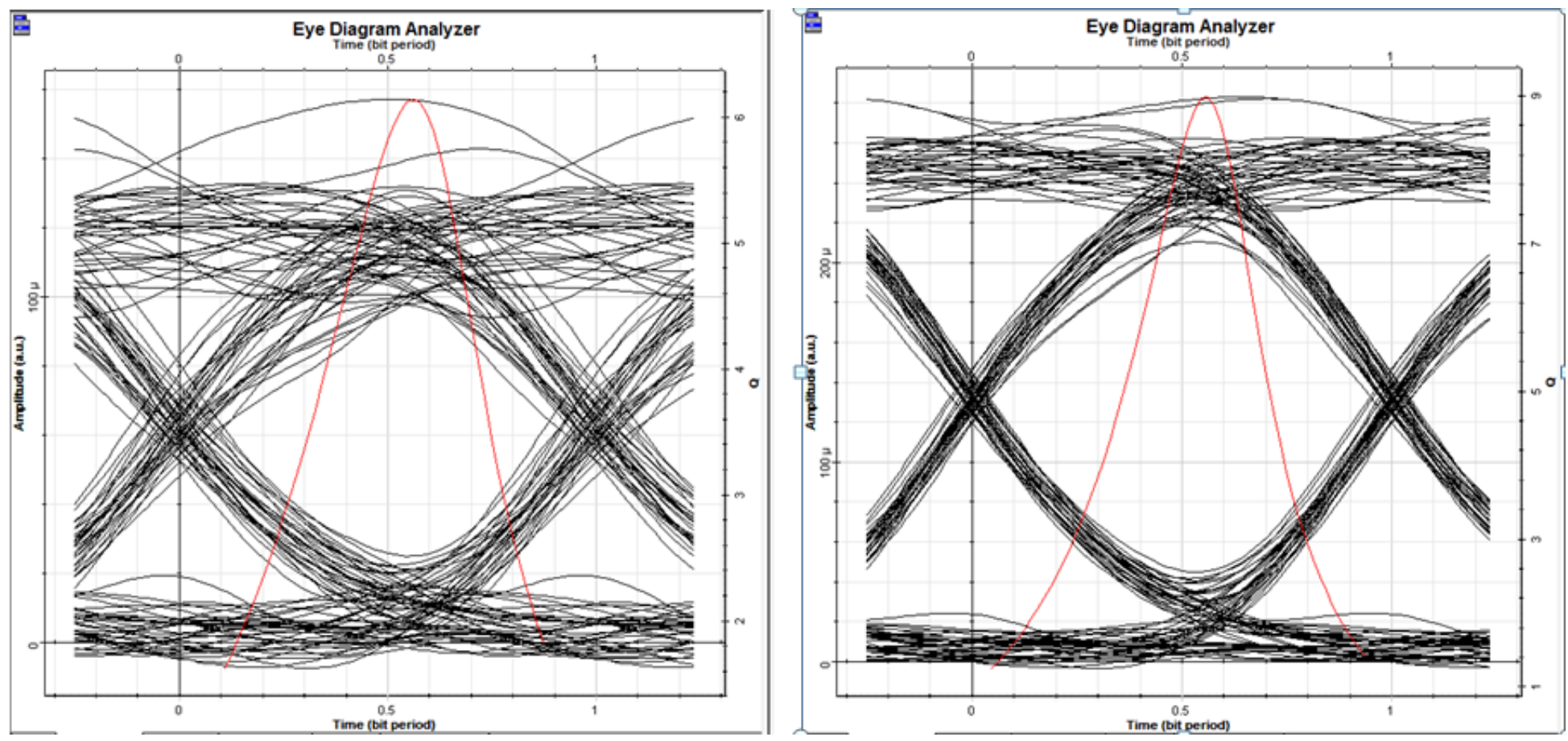

Figure 5

Eye diagrams of Single beam \& Multibeam WDM FSO system using Homodyne detection at $10 \mathrm{~km}$ under scintillation effect

\section{Link distance versus $Q$-factor}

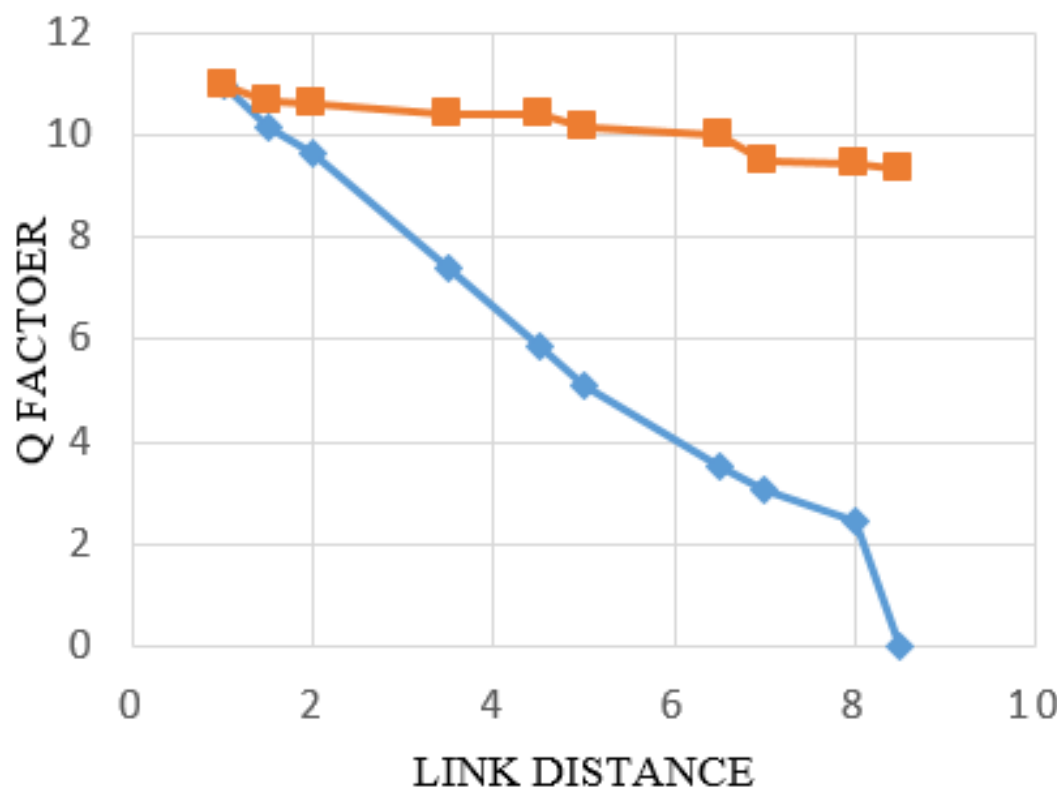

Figure 6 
Comparison of Multibeam WDM-FSO system using direct detection and Multibeam WDM-FSO system using Homodyne detection.
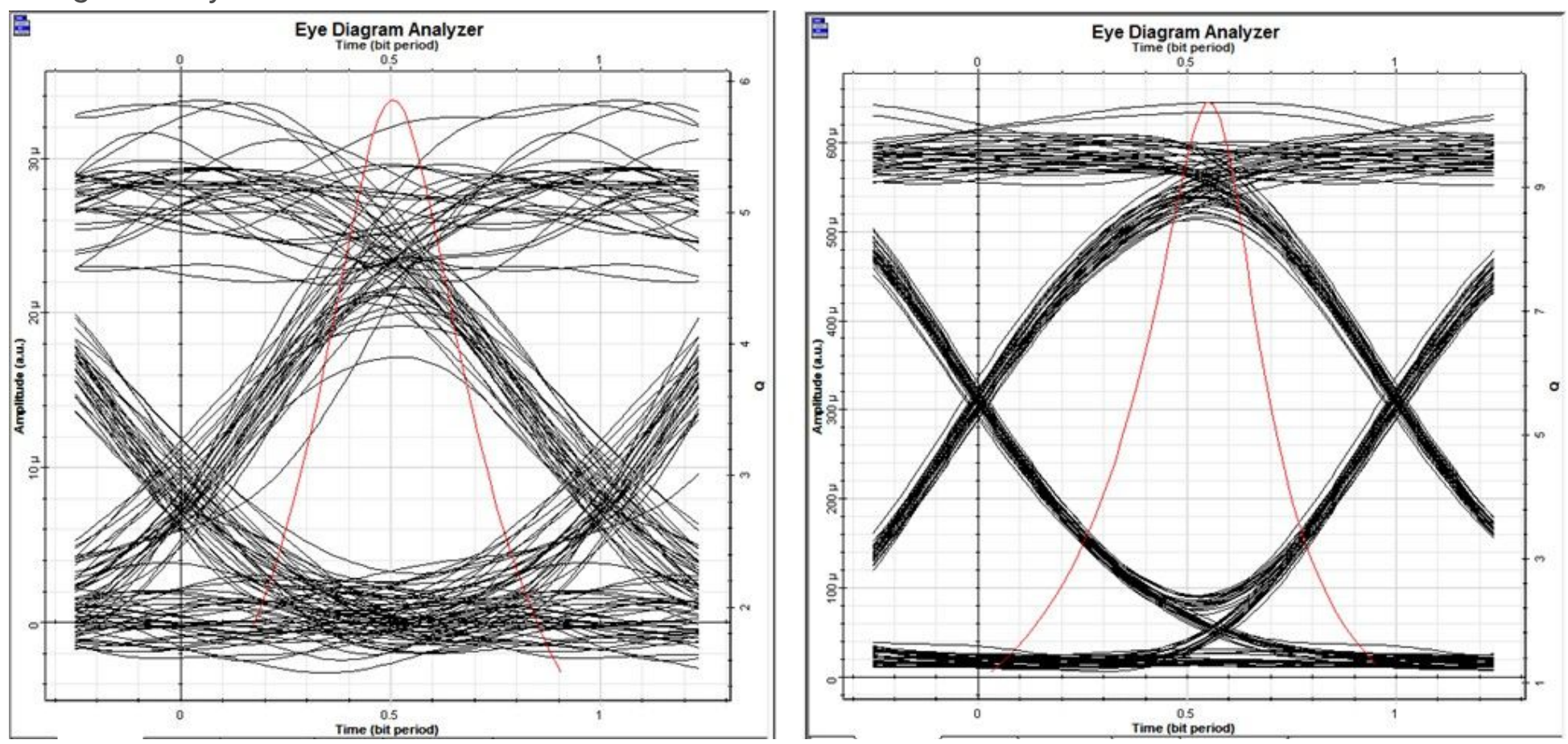

Figure 7

Eye diagrams comparing Multibeam WDM-FSO system (a) using direct detection with the similar system (b) using Homodyne detection at a distance of $4.5 \mathrm{~km}$.

\section{Input laser power versus Quality factor}

\section{(dBm)}

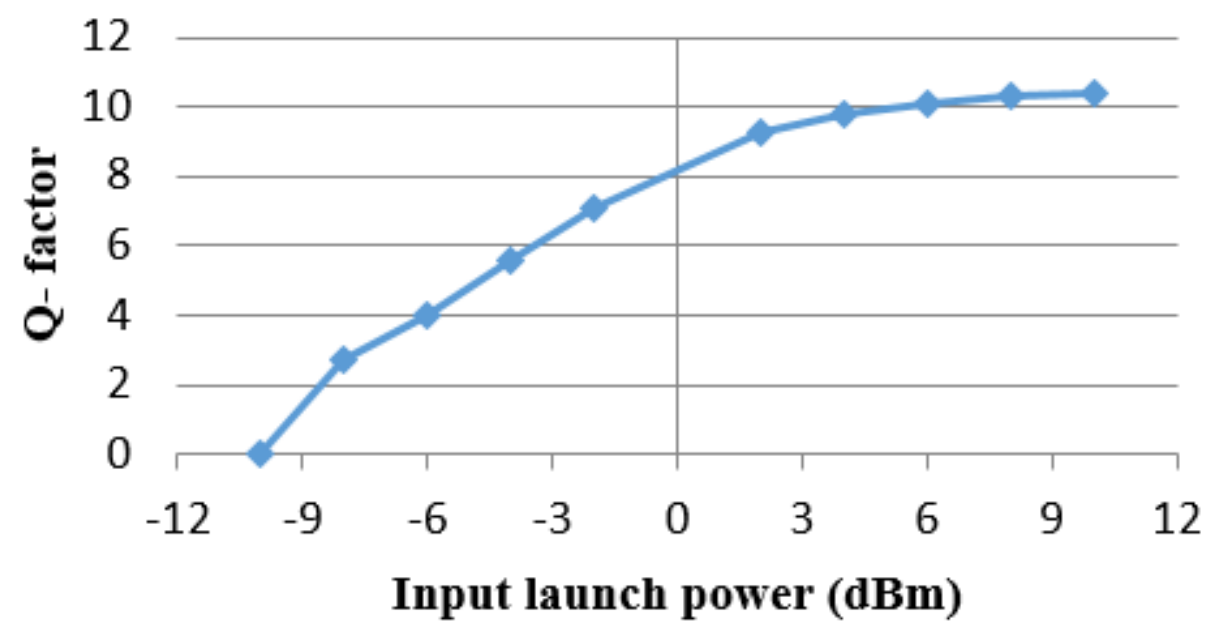

Figure 8

Input laser power versus Q-factor at distance of $4.5 \mathrm{~km}$ with data rate of $10 \mathrm{Gbps}$ 


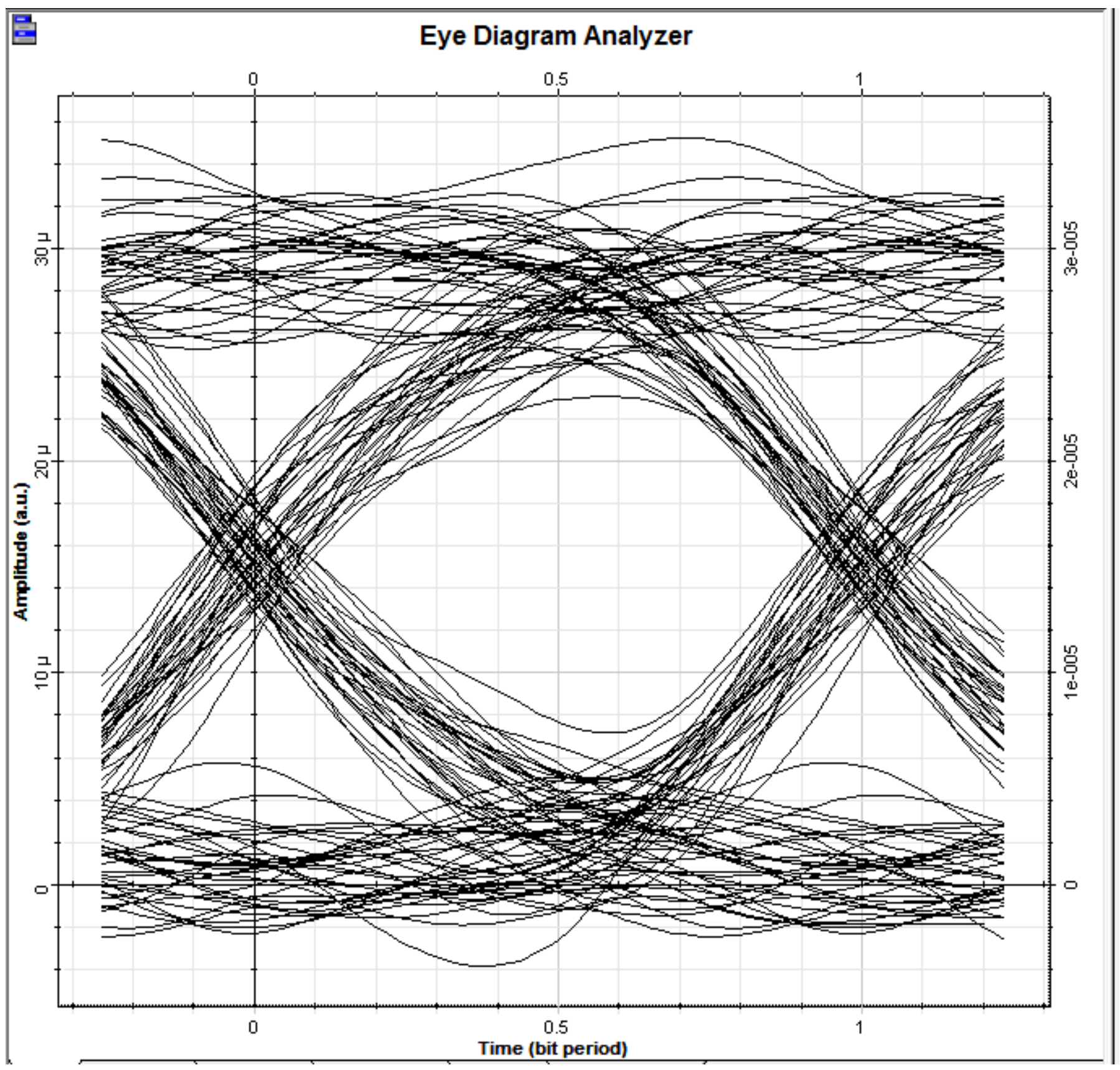

Figure 9

Eye diagram of the proposed Multibeam WDM-FSO system using Homodyne detection at $4.5 \mathrm{~km}$ at $10 \mathrm{~km}$ under scintillation effect 